MATHEMATICS OF COMPUTATION

65 , Number 214

April 1996, Pages 861-875

\title{
ON THE BETA EXPANSION FOR SALEM NUMBERS OF DEGREE 6
}

\author{
DAVID W. BOYD
}

\begin{abstract}
For a given $\beta>1$, the beta transformation $T=T_{\beta}$ is defined for $x \in[0,1]$ by $T x:=\beta x(\bmod 1)$. The number $\beta$ is said to be a beta number if the orbit $\left\{T^{n}(1)\right\}_{n \geq 1}$ is finite, hence eventually periodic. It is known that all Pisot numbers are beta numbers, and it is conjectured that this is true for Salem numbers, but this is known only for Salem numbers of degree 4 . Here we consider some computational and heuristic evidence for the conjecture in the case of Salem numbers of degree 6, by considering the set of 11836 such numbers of trace at most 15. Although the orbit is small for the majority of these numbers, there are some examples for which the orbit size is shown to exceed $10^{9}$ and for which the possibility remains that the orbit is infinite. There are also some very large orbits which have been shown to be finite: an example is given for which the preperiod length is 39420662 and the period length is 93218808 . This is in contrast to Salem numbers of degree 4 where the orbit size is bounded by $2 \beta+3$. An heuristic probabilistic model is proposed which explains the difference between the degree- 4 and degree- 6 cases. The model predicts that all Salem numbers of degree 4 and 6 should be beta numbers but that degree-6 Salem numbers can have orbits which are arbitrarily large relative to the size of $\beta$. Furthermore, the model predicts that a positive proportion of Salem numbers of any fixed degree $\geq 8$ will not be beta numbers. This latter prediction is not tested here.
\end{abstract}

\section{Introduction}

The purpose of this paper is to present some results and observations regarding the beta transformations introduced by Rényi [8]. Given $\beta>1$, the beta transformation $T=T_{\beta}$ is defined for $x \in[0,1]$ by $T x:=\beta x \bmod 1$. Parry [7] defined $\beta$ to be a beta number if the orbit $\left\{T^{n}(1)\right\}$ is finite. If $T^{n}(1)=0$ for some $n$, then $\beta$ is a simple beta number. If $\beta$ is a beta number which is not simple, then there is some smallest $m \geq 1$ (the preperiod length) and $p \geq 1$ (the period length) for which $T^{m+p}(1)=T^{m}(1)$. For a simple beta number we define $m=0$ and $p$ to be the smallest integer with $T^{p}(1)=0$. Notice that $m+p$ is the size of the orbit $\left\{T^{n}(1)\right\}_{n \geq 1}$.

It is easy to see by induction (see below) that there are integers $c_{k}$ with $0 \leq c_{k}<$ $\beta$ (the digits in the beta expansion of 1 ) for which $T^{n}(1)=\beta^{n}-c_{1} \beta^{n-1}-\cdots-c_{n}$, so that $T^{n}(1)=P_{n}(\beta)$, where

$$
P_{n}(x)=x^{n}-c_{1} x^{n-1}-\cdots-c_{n} .
$$

Received by the editor June 20, 1994.

1991 Mathematics Subject Classification. Primary 11R06, 11K16; Secondary 11Y99.

Key words and phrases. Salem numbers, beta expansions, polynomials, computation.

This research was supported by a grant from NSERC. 
Thus, if $\beta$ is a beta number, then $\beta$ satisfies the polynomial equation $R(\beta)=0$, where

$$
R(x)= \begin{cases}P_{m+p}(x)-P_{m}(x) & \text { if } m>0 \\ P_{p}(x) & \text { if } m=0\end{cases}
$$

In particular, a beta number is an algebraic integer whose minimal polynomial $P(x)$ divides $R(x)$. The degree $D=D(\beta):=m+p$ of $R$ is the beta degree of $\beta$, which is in general larger than $\operatorname{deg}(\beta)$, the degree of $\beta$, which is defined to be the degree of its minimal polynomial $P$. As we observed above, $D$ is the size of the orbit $\left\{T^{n}(1)\right\}_{n \geq 1}$.

The polynomial $R$ is called the characteristic polynomial of $\beta$. Parry [7] showed that the roots of $R$ other than $\beta$ lie in the disk $|z|<\min (2, \beta)$. This was improved to $|z| \leq(\sqrt{5}+1) / 2$ by Solomyak [11] and Flatto, Lagarias and Poonen [4] independently. In fact, Solomyak gives an exact description of the compact subset of the plane which is the closure of the set of all conjugates (other than $\beta$ ) of beta numbers.

Recall that a Pisot number (or Pisot-Vijayaraghavan number, or PV number) is an algebraic integer $\beta>1$ for which all conjugates $\gamma$ of $\beta$ with $\gamma \neq \beta$ satisfy $|\gamma|<1$. A Salem number is an algebraic integer $\beta>1$ for which all conjugates $\gamma \neq \beta$ satisfy $|\gamma| \leq 1$ with at least one conjugate having $|\gamma|=1$. This implies that $\beta$ is reciprocal, so $\beta^{-1}$ is a conjugate of $\beta$ and all other conjugates $\gamma$ satisfy $|\gamma|=1$. Hence, $\operatorname{deg}(\beta)$ is even and $\geq 4[9, \mathrm{p} .26]$.

Schmidt [10] showed that every Pisot number is a beta number. This was also proved independently by Bertrand [1]. The idea behind the proof, which is based on the box principle, goes back to a paper of Gelfond [5]. Schmidt also showed that if every rational $x \in[0,1]$ has a periodic expansion in base $\beta$ (i.e., the orbit $\left\{T^{n}(x)\right\}$ is finite), then $\beta$ must be a Pisot or Salem number. He conjectured that the converse is true, so in particular that every Salem number would be a beta number. A simple result from [3] is that a Salem number cannot be a simple beta number: clearly the characteristic polynomial of a simple beta number has a unique positive root, but a Salem number $\beta$ has two positive conjugates, namely $\beta$ and $1 / \beta$.

In [3], we showed that if $\beta$ is a Salem number of degree 4 , then $\beta$ is a beta number. (We did not consider Schmidt's more general conjecture there; it remains open for Salem numbers of all degrees.) The explicit beta expansion of each such $\beta$ was given. In all cases $m=1$, i.e., $\beta(\bmod 1)$ is a purely periodic point of $T$. If $\beta$ has the minimal polynomial $P(x)=x^{4}-a x^{3}+b x^{2}-a x+1$, then for fixed $a$ the period $p=p(b)$ is a unimodal function of $b$ and takes on values which lie in the set $\{3,5,9\} \cup\{p$ even : $6 \leq p \leq 2 a-4\}$. Thus, $D=m+p \leq 2 a-3<2 a=2 \operatorname{trace}(\beta)$ for all Salem numbers of degree 4 . No such bound seems to be true for Salem numbers of higher degree.

If we write $\alpha_{n}=T^{n}(1)$, then the canonical beta expansion of 1 to base $\beta>1$ is defined by the "greedy" algorithm: $\alpha_{0}=1, c_{n}=\left\lfloor\beta \alpha_{n-1}\right\rfloor$ and $\alpha_{n}=\beta \alpha_{n-1}-c_{n}$, for $n \geq 1$. Clearly, $0 \leq c_{n}<\beta$ for all $n$, and

$$
1=\sum_{n=1}^{\infty} c_{n} \beta^{-n}
$$


We will refer to the sequence $\left\{c_{n}\right\}$ as the beta expansion (of 1 ) for $\beta$. If this expansion is periodic, with preperiod length $m$ and period length $p$, we denote it by $c_{1} \ldots c_{p}$, if $m=0$, and by $c_{1} \ldots c_{m}: c_{m+1} \ldots c_{m+p}$ if $m>0$. A simple criterion for $\left\{c_{n}\right\}$ to be the beta expansion for some $\beta>1$ was given by Parry [7,p.407]: the sequence $c_{1}, c_{2}, \ldots$ must dominate, in the sense of lexicographic order, the shifted sequences $c_{k}, c_{k+1}, \ldots$, for all $k \geq 1$. The number $\beta$ is then determined by (1.3). For periodic expansions, $\beta$ is also determined by (1.2).

Our main purpose here is to consider whether Salem numbers of degree 6 must be beta numbers. We compute the beta expansions for all but 80 of the Salem numbers of degree 6 and trace at most 15 (there are 11836 such numbers). For all but 199 of these, we have $\max (m, p)<1000$. However, there is one example for which $(m, p)=(39420662,93218808)$, and two examples for which we can show that $D>10^{9}$ but do not know whether $D<\infty$. The existence of such examples shows that the situation for Salem numbers of degree 6 is certainly more complicated than the degree- 4 case. However, the fact that there are examples with $D$ very large but finite perhaps supports Schmidt's conjecture for Salem numbers of degree 6 .

In $\S 6$, we give a heuristic probabilistic argument based on ideas of the geometry of numbers which makes plausible the observed difference between the cases $\operatorname{deg}(\beta)=$ 4 and $\operatorname{deg}(\beta)=6$. The argument correctly predicts that $D(\beta)$ will be small relative to $\beta$ for Salem numbers of degree 4 . It predicts that almost all Salem numbers of degree 6 will be beta numbers but that $D(\beta)$ can be arbitrarily large even for small $\beta$. Moreover, it predicts that for each fixed even degree $d \geq 8$ there should be a positive proportion of Salem numbers of degree $d$ which are not beta numbers, as well as a positive proportion that are beta numbers. We present no computational evidence here for this latter prediction.

The heuristic argument suggests, for Salem numbers of fixed degree $d$, that the size of $D(\beta)$ is directly related to the size of $\beta^{d-1} /|\operatorname{disc}(\beta)|^{1 / 2}$. Here $\operatorname{disc}(\beta)$ denotes the discriminant of the polynomial $P$. We explore this connection through some of the computed examples for $d=6$.

The heuristic arguments apply equally well to Schmidt's more general conjecture concerning the periodicity of the beta expansions of rationals to a Salem number base, and predict that Schmidt's conjecture is true for numbers of degree 4 or 6 and false for numbers of higher degree. For $d=4$, the model predicts that the size of the orbit $\left\{T^{n}(p / q)\right\}$ for fixed $\beta$ should be roughly proportional to $q^{4}$. These predictions are not tested here.

\section{Recognizing SAlem numbers of Degree 6}

A Salem number $\beta$ of degree 6 has a minimal polynomial of the following form:

$$
P(x):=x^{6}+a x^{5}+b x^{4}+c x^{3}+b x^{2}+a x+1,
$$

where $a, b$ and $c$ are integers. The trace of $\beta$ is $\operatorname{trace}(\beta)=-a=\sum_{i} \beta_{i}$, where the sum is over the six conjugates of $\beta$, i.e., the six roots of (2.1). Two of these conjugates are $\beta$ and $1 / \beta$ and the remaining conjugates satisfy $\left|\beta_{i}\right|=1$, so a bound on $|a|$ implies a bound on $\beta$ and hence on $|b|$ and $|c|$. So, for fixed $a$, there is a finite set of $\beta$ with trace $(\beta)=-a$. Observe that

$$
\operatorname{trace}(\beta)=-a>\beta+1 / \beta-\sum_{i=3}^{6}\left|\beta_{i}\right|>2-4=-2,
$$


so $\operatorname{trace}(\beta) \geq-1$ for all Salem numbers of degree 6 . In fact, there are no such numbers with trace $-1,4$ with trace 0,15 with trace 1 , and 39 with trace 2 .

It is not difficult to recognize Salem numbers of degree 6 . Since $P(x)$ is reciprocal, we can write

$$
P(x)=x^{3} U(x+1 / x)
$$

where

$$
U(x)=x^{3}+a x^{2}+(b-3) x+(c-2 a) .
$$

The zeros of $U$ are the numbers $\beta_{i}+1 / \beta_{i}$ and hence $U$ must have two roots in the open interval $-2<x<2$ and one root with $x>2$. This is equivalent to the following requirements: (i) $U( \pm 2)<0$, (ii) $U^{\prime}(x)$ has real roots, the smaller of which, $\xi_{1}$ satisfies $-2<\xi_{1}<2$, and $U\left(\xi_{1}\right)>0$. We used this criterion in compiling our list of Salem numbers.

We also require $U$ to be irreducible. Since $U$ is cubic, it suffices that $U(n) \neq 0$ for any integer $n$. Only a finite set of $n$ need be checked since, by a well-known estimate of Cauchy, $U(x) \neq 0$ for $|x| \geq 1+\max (|a|,|b-3|,|c-2 a|)$. It is easy to see that $P$ can only factor into factors of even degree since the roots $\beta$ and $1 / \beta$ must belong to the same factor, or else there would be a factor the product of whose roots is in absolute value less than 1 , which is clearly impossible. Thus, $P$ is irreducible if and only if $U$ is irreducible.

The following useful result is an elementary deduction from the above discussion.

Lemma 2.1. Let $P$ be as in (2.1) and $U$ as in (2.3). A necessary condition for $P$ to be the minimal polynomial of a Salem number is that

$$
U( \pm 2)<0 \quad \text { and } \quad U(n) \neq 0
$$

for $-1 \leq n \leq 1+\max (|a|,|b-3|,|c-2 a|)$. A sufficient condition for $P$ to be the minimal polynomial of a Salem number is, in addition to (2.4), any one of $U(-1)>0, U(0)>0$ or $U(1)>0$.

In general, if $P$ is the minimal polynomial of a Salem number of degree $d=2 s$, then there is a monic polynomial $U$ with integer coefficients for which $P(x)=$ $x^{s} U(x+1 / x)$. The numbers $\beta_{i}+1 / \beta_{i}$ are the zeros of $U$ repeated twice each. The following computation gives a useful expression for $\operatorname{disc}(\beta)$ :

$$
\begin{aligned}
\operatorname{disc}(P) & =(-1)^{\left(\begin{array}{c}
d \\
2
\end{array}\right)} \prod_{i} P^{\prime}\left(\beta_{i}\right)=(-1)^{s} \prod_{i} \beta_{i}^{s} U^{\prime}\left(\beta_{i}+1 / \beta_{i}\right)\left(1-\beta_{i}^{-2}\right) \\
& =(-1)^{s} P(1) P(-1) \operatorname{disc}(U)^{2}=U(2) U(-2) \operatorname{disc}(U)^{2}
\end{aligned}
$$

\section{Are SAlem numbers of Degree 6 Beta numbers?}

In this section we describe the results of a computation of the beta expansions for the 11836 Salem numbers of degree 6 and trace at most 15. The complete expansion was obtained except in 80 cases. The distribution of the pairs $(m, p)$ for these numbers is quite remarkable. For the most part there is a great deal of regularity, and the values of $m$ and $p$ are quite small: for all but 199 of the numbers 
TABLE $3(a=-3$ and $b=-1)$

\begin{tabular}{ccccccc}
$c$ & $m$ & $p$ & $\operatorname{disc}(\beta)$ & $\operatorname{disc}(\beta)$ & $\beta$ & $C(\beta)^{\dagger}$ \\
\hline \multicolumn{7}{c}{} \\
5 & 1 & 12 & 485809 & $17^{2} \cdot 41^{2}$ & 2.81016 & .0689 \\
4 & 1 & 9 & 3534400 & $2^{6} \cdot 5^{2} \cdot 47^{2}$ & 2.95386 & .0328 \\
3 & 1 & 7 & 11471769 & $3^{2} \cdot 1129^{2}$ & 3.07216 & .0222 \\
2 & 1 & 7 & 25563136 & $2^{12} \cdot 79^{2}$ & 3.17425 & .0175 \\
1 & 1 & 7 & 45225625 & $5^{4} \cdot 269^{2}$ & 3.26491 & .0151 \\
-1 & 1 & 5 & 88642225 & $5^{2} \cdot 7^{2} \cdot 269^{2}$ & 3.42227 & .0137 \\
-2 & 1 & 5 & 102252544 & $2^{14} \cdot 79^{2}$ & 3.49209 & .0141 \\
-3 & 1 & 5 & 103245921 & $3^{4} \cdot 1129^{2}$ & 3.55736 & .0154 \\
-4 & 1 & 14 & 88360000 & $2^{6} \cdot 5^{4} \cdot 47^{2}$ & 3.61877 & .0181 \\
-5 & 1 & 12 & 58782889 & $11^{2} \cdot 17^{2} \cdot 41^{2}$ & 3.67857 & .0240 \\
-7 & $*$ & $*$ & 405769 & $7^{4} \cdot 13^{2}$ & 3.78470 & .3342 \\
\multicolumn{7}{c}{} \\
$*$ & for $c=-7, m+p>1199978517$. \\
\end{tabular}

surveyed, both $m<1000$ and $p<1000$. For 9609 of the numbers ( $81 \%$ of the total) we have $m=1$, but, in contrast to the degree- 4 situation, larger values of $m$ do occur with a certain regularity. However, among the remaining 199 cases, there are at least 79 for which $D=m+p>10^{6}$ and at least two for which $D>10^{9}$.

The two known examples with $D>10^{9}$ are $(a, b, c)=(-3,-1,-7)$ and $(a, b, c)=$ $(-5,-2,-11)$. This might suggest that these $\beta$ are not beta numbers. However, there are some very large values of $m$ and $p$ among the cases where $(m, p)$ has been determined. The (current) record is attained for $(-6,-5,-14)$, which has $(m, p)=(39420662,93218808)$. Other notable values are $(-5,-22,-33)$ with $(m, p)=(8604828,9101)$, and $(-9,-23,-28)$ with $(m, p)=(1979174,11754)$.

A sample of these numerical results is presented in two tables organized as follows. In Table 1 of the Supplement all quintuples $(a, b, c, m, p)$ with $0 \leq-a=$ $\operatorname{trace}(\beta) \leq 5$ are listed, provided both $m<10000$ and $p<10000$. The complete table for $0 \leq \operatorname{trace}(\beta) \leq 15$ is over 20 times longer than that presented here and has much the same general appearance. Table 2 of the Supplement gives the 36 values $(a, b, c)$ with $-a \leq 10$ for which $\min (m, p) \geq 10000$. This includes 18 cases where only a lower bound for $D=m+p$ is known.

Although there must be other factors involved, the argument of $\S 6$ suggests that the size of $D(\beta)$ is directly related to the size of $C(\beta)=(\pi / 6)^{2} \beta^{d-1} / \operatorname{disc}(\beta)^{1 / 2}$. In particular, numbers with smaller discriminants should have larger orbits. The prediction of $\S 6.6$ is that the orbit will be finite provided $C(\beta)<1$. We illustrate this in Table 3, where we list the values of $m, p, \operatorname{disc}(\beta)$ and $C(\beta)$ for the 7 Salem numbers with $a=-3, b=-1$, so $-7 \leq c \leq 5$. The omitted values of $c$ correspond to reducible polynomials.

One might expect that the size of the period of $\beta$ might depend on arithmetic properties of $\beta$ or perhaps on the existence of approximate multiplicative relationships between the conjugates $\gamma$ of $\beta$ with $|\gamma|=1$. If this were true, then one would expect powers of $\beta$ to behave in a manner similar to $\beta$. A counterexample to this expectation is the $\beta$ with $(a, b, c)=(-3,-1,-7)$, where $D>10^{9}$. Here, $\beta^{2}$ has $(a, b, c)=(-11,-43,-63)$ with $(m, p)=(1,102)$. 
TABLE 4 (powers of $\beta=1.40126837 \ldots$ )

\begin{tabular}{cccccccccc}
$k$ & $a$ & $b$ & $c$ & $m$ & $p$ & $\operatorname{disc}(\beta)$ & $\operatorname{disc}(\beta)$ & $\beta^{k}$ & $C\left(\beta^{k}\right)^{\dagger}$ \\
\hline 1 & 0 & -1 & -1 & 1 & 7 & 52441 & $229^{2}$ & 1.40127 & .0065 \\
2 & -2 & -1 & 3 & 1 & 9 & 52441 & $229^{2}$ & 1.96355 & .0349 \\
3 & -3 & 2 & -4 & 1 & 10 & 3356224 & $2^{6} \cdot 229^{2}$ & 2.75146 & .0236 \\
4 & -6 & 11 & -13 & 1 & 22 & 2569609 & $7^{2} \cdot 229^{2}$ & 3.85554 & .1457 \\
5 & -5 & -1 & -6 & 1 & 14 & 3436773376 & $2^{16} \cdot 229^{2}$ & 5.40265 & .0215 \\
6 & -5 & -16 & -24 & 8867 & 439 & 13424896 & $2^{8} \cdot 229^{2}$ & 7.57056 & 1.8607 \\
7 & -14 & 41 & -57 & $*$ & $*$ & 8862529 & $13^{2} \cdot 229^{2}$ & 10.60838 & 12.3727 \\
8 & -14 & -13 & 3 & 131 & 267 & 12538872214729 & $7^{2} \cdot 47^{4} \cdot 229^{2}$ & 14.86519 & .0562 \\
\multicolumn{7}{c}{} & & & \\
\end{tabular}

Exploring this theme further, we consider the powers of the smallest Salem number of degree $6, \beta=1.40126 \ldots$, which has $(a, b, c)=(0,-1,-1)$. For $1 \leq k \leq 8$, Table 4 gives $(a, b, c)$ for the minimal polynomial of $\beta^{k}$, the values of $m$ and $p$, $\operatorname{disc}\left(\beta^{k}\right)$ and $C\left(\beta^{k}\right)$. The factored form of $\operatorname{disc}(\beta)$ is given for the sake of interest, although the arithmetic properties of $\beta$ appear to play no role here.

\section{Patterns AND Regularities in the tables}

Many regularities are apparent in Table 1. For example, it is easy to spot numerous occurrences of $(m, p)=(1,5),(1,6),(1,7),(5,33)$, etc., and even to guess the general pattern of such occurrences. Such results can usually be proved by the method of [3]. That is, one guesses the expansion $c_{1} \ldots c_{m}: c_{m+1} \ldots c_{m+p}$ of $\beta$, verifies that this purported expansion is a legitimate expansion of a beta number $\beta^{\prime}$ according to Parry's criterion, computes the characteristic polynomial $R(x)$ of $\beta^{\prime}$ by (1.2), and verifies that $R(x)$ is divisible by $P(x)$, the minimal polynomial of $\beta$. For example, here are some simple cases:

Proposition 4.1. Let $\beta$ be a Salem number of degree 6 with minimal polynomial $P(x)$ given by (2.1). Then $(m, p)=(1,5)$ if and only if $a \leq b \leq 0$ and $a \leq c \leq 0$ and $a \leq-1$.

Proof. For convenience, write $(a, b, c)=(-A,-B,-C)$. The minimal polynomial $P(x)$ of $\beta$ must satisfy

$$
P(x)=P_{6}(x)-P_{1}(x)
$$

This implies that

$$
c_{1}: c_{2}, \ldots, c_{6}=A: B, C, B, A-1, A-1 .
$$

Next we must determine the conditions under which the expansion satisfies $c_{n} \geq 0$, for all $n$, and that, for all $n>1$,

$$
\left(c_{1}, c_{2}, \ldots\right)>\left(c_{n}, c_{n+1}, \ldots\right),
$$

where, in (4.3), $>$ denotes lexicographical order [7,p.407]. From (4.2), we see that the condition $c_{1} \geq c_{n} \geq 0$, for all $n$, implies $A \geq 1, A \geq B \geq 0$, and $A \geq C \geq 0$. 
But it is easily seen that these conditions insure that (4.3) holds for all $n$ and hence that $\left(c_{1}, c_{2}, \ldots\right)$ is the beta expansion for a number $\beta^{\prime}$ given by (1.3).

The right member of (4.2) defines a sequence with $m=1$ and $p$ a divisor of 5 , so either $p=1$ or 5 . However, $p=1$ occurs only if $B=C=A-1$ and this is the expansion of a beta with minimal polynomial $x^{2}-(A+1) x+1$, not a Salem number. This can be easily seen from (1.3) or by observing that the polynomial $U(x)=x^{3}-A x^{2}-(A+2) x+(A+1)$ vanishes at $x=A+1$.

The periodicity of $\left\{c_{n}\right\}$ shows that $R(x)=P_{6}(x)-P_{1}(x)$ is the characteristic polynomial of $\beta^{\prime}$. But (4.1) shows that $R(x)=P(x)$, the minimal polynomial of $\beta$. Hence, $\beta=\beta^{\prime}$, so $\beta$ has $(m, p)=(1,5)$.

Proposition 4.2. Let $\beta$ be a Salem number of degree 6 with minimal polynomial $P(x)$ given by $(2.1)$. Then $(m, p)=(1,6)$ if and only if $(a, b, c)=(-A, B,-C)$, where $A, B$ and $C$ are positive integers satisfying $A \geq 2, A \geq B, A+B-1 \geq C \geq$ $B \geq 1$ with the further condition that if $A+B-1=C$, then $A=C$ and $B=1$.

Proof. Suppose the beta expansion of $\beta$ is $c_{1}: c_{2} \ldots c_{7}$, so that the characteristic polynomial is

$$
R(x)=P_{7}(x)-P_{1}(x)=x^{7}-c_{1} x^{6}-\cdots-c_{5} x^{2}-\left(c_{6}+1\right) x-\left(c_{7}-c_{1}\right) .
$$

Let $P(x)=x^{6}-A x^{5}+B x^{4}-C x^{3}+B x^{2}-A x+1$ be the minimal polynomial of $\beta$. If this is to divide $R(x)$, then the quotient must be of the form $x+d$ for some integer $d \neq 0$. By a result of Parry mentioned earlier, since $-d$ is a root of $R(x)$ other than $\beta$, it must satisfy $|d|<2$ and hence $d= \pm 1$. From (4.4), $d=c_{1}-c_{7}$, and since $c_{1} \geq c_{7}$ by Parry's criterion, we must have $d=1$. Equating the coefficients of $P(x)(x+1)$ with those of $(4.4)$ thus shows that

$$
c_{1}: c_{2}, \ldots, c_{7}=A-1: A-B, C-B, C-B, A-B, A-2, A-2 .
$$

The conditions $c_{n} \geq 0$ for all $n$ imply $A \geq 2, A \geq B$ and $C \geq B$. The conditions $c_{1} \geq c_{2}$ and $c_{1} \geq c_{3}$ imply $B \geq 1$ and $A+B-1 \geq C$. Finally, if $c_{1}=c_{3}$, i.e., $C=A+B-1$, then we must have $c_{2} \geq c_{4}$, that is $A \geq C$; but $B \geq 1$ implies $C=A+B-1 \geq A$, so in this case $A=\bar{C}$ and hence $B=1$.

To finish the proof, we must verify that Parry's criterion follows from the inequalities in the statement of the proposition, the equation (4.5) defining $c_{n}$ for $n \leq 7$, and the assumed periodicity of $c_{n}$. This involves checking only a finite number of cases: for example, to verify that $\left(c_{1}, c_{2}, \ldots\right) \geq\left(c_{4}, c_{5}, \ldots\right)$, we note that $c_{1} \geq c_{4}$ follows from $A+B-1 \geq C$, and that if $c_{1}=c_{4}$, then $A=C$ and $B=1$, so $c_{2}=c_{5}$ (always true here), and $c_{3}=C-B=A-1>A-2=c_{6}$. The remaining cases are similar and left to the reader.

Finally, we must verify that the exact period is 6 and not a divisor of 6 . For example, if $p=1$, then (4.5) would imply that $A=C$ and $B=2$. Then we find that $U(x)=x^{3}-A x^{2}-x+A$, so that $U(1)=0$ and hence $P$ is reducible. Similarly, $p=2$ or $p=3$ lead to $U(1)=0$ and the reducibility of $P$.

Proposition 4.3. Let $\beta$ be a Salem number of degree 6 for which

$$
(a, b, c)=(-A+1,-A,-2 A)
$$

for some $A \geq 4$. Then $(m, p)=(5,33)$. 
Proof. Let $P_{A}(x)=x^{6}+x^{5}+x+1-A\left(x^{5}+x^{4}+2 x^{3}+x^{2}+x\right)$. Then $P_{A}$ is the minimal polynomial of a Salem number $\beta_{A}$, for $A>0$, by Lemma 2.1. Let $F_{q}(x)$ denote the $q$ th cyclotomic polynomial (the minimal polynomial of the primitive $q$ th roots of unity), and define $Q(x)=F_{2}(x) F_{6}(x) F_{33}(x)\left(x^{9}+x^{2}-1\right)$, so that $Q$ is a nonreciprocal polynomial of degree 32 . We claim that the beta expansion of $\beta_{A}$ has $(m, p)=(5,33)$ and is given explicitly by

(4.6)

$$
\begin{gathered}
A, 1,(A-2), A, 1:(A-2), 0, A, 0,0,(A-2), 1,1,(A-2), 0,0, A, 0,(A-2), 2,0 \\
(A-4), 3,1,(A-4), 2,0,(A-2), 2,(A-2), 0,2,(A-4), 1,3,(A-4), 0,2 .
\end{gathered}
$$

To prove this, we note that, for $A \geq 4$, the sequence $\left\{c_{n}\right\}$ defined by (4.6) is the beta expansion of some $\beta_{A}^{\prime}$, since it satisfies Parry's criterion. This is obvious since $c_{1}=A \geq c_{n}$ for all $n$ and if equality holds (i.e., $n=8$ or 17 ), then $c_{2}=1>c_{n+1}=$ 0 . The condition $A \geq 4$ guarantees $c_{n} \geq 0$ for all $n$.

Let $R_{A}(x)$ be the characteristic polynomial of $\beta_{A}^{\prime}$. We must next verify that

$$
P_{A}(x) Q(x)=R_{A}(x)
$$

This is an elementary algebraic computation which is made easier by observing that the coefficients of $P_{A}(x)$ and $R_{A}(x)$ are linear functions of $A$, and hence the identity need only be established for two values of $A$, e.g. $A=0$ and $A=1$. We leave this to the reader who may wish to use a computer algebra system.

Since $Q(x)$ has no positive roots, (4.7) implies that $\beta_{A}=\beta_{A}^{\prime}$, and hence that (4.6) is the beta expansion of $\beta_{A}$. It is clear in this case that the period of the expansion is 33 and not a proper divisor of 33 because of the positions of the two $c_{n}=A$.

Remark 4.1. The condition in Proposition 4.3 is only a sufficient condition for $(m, p)=(5,33)$. There are many other occurrences of $(5,33)$ besides the ones described here.

\section{THE COMPUTATION OF THE BETA EXPANSION}

5.1. The basic algorithm. Recalling the definitions of $\S 1$, we write $\alpha_{n}=T^{n}(1)$ for $n \geq 0$, and $c_{n}=\left\lfloor\beta \alpha_{n-1}\right\rfloor$ for $n \geq 1$. Consider first how one would compute these quantities using an approximation to the real number $\beta$. If an approximation $\beta_{0}$ to $\beta$ is used which has $\left|\beta-\beta_{0}\right|=\epsilon$, then the error in the corresponding approximation to $\alpha_{n}$ is about $\beta^{n} \epsilon$. Thus, for large $n$ it will be impossible to compute $c_{n}$ correctly. In addition, even for small $n$, it would be impossible to decide whether or not $\alpha_{m}=\alpha_{n}$ for some $m \neq n$.

On the other hand, if $\beta$ is an algebraic integer, then all $\alpha_{n}$ lie in $\mathbb{Z}[\beta]$. Specifically, if $\beta$ has degree $d$ and minimal polynomial $P$, and if $B_{n}$ is the polynomial of degree $d-1$ defined by $B_{n}(x) \equiv P_{n}(x)(\bmod P(x))$, where $P_{n}(x)$ is as in $\S 1$, then $\alpha_{n}=$ $B_{n}(\beta)$. The $B_{n}$ thus provide an exact representation of $\alpha_{n}$, so the question $\alpha_{n}=\alpha_{m}$ can be effectively answered. Since the $B_{n}$ satisfy the recurrence

$$
B_{n}(x) \equiv x B_{n-1}(x)-c_{n} \quad(\bmod P(x))
$$


the computation simply involves a shift of the coefficients of $B_{n-1}$ followed by the replacement of $x^{d}$ by $x^{d}-P(x)$.

The only difficulty is the determination of $c_{n}=\left\lfloor\beta B_{n-1}(\beta)\right\rfloor$. This can be computed using an approximation $\beta_{0}$ to $\beta$ of modest accuracy unless $\beta \alpha_{n-1}$ is unusually close to an integer $c$. If $\beta$ is a Salem number, standard arguments from transcendence theory show that

$$
\left|\beta \alpha_{n-1}-c\right|>\left(L\left(B_{n-1}\right)+c_{1}+1\right)^{-d+1},
$$

where $L\left(B_{n-1}\right)$ denotes the sum of the absolute values of the coefficients of $B_{n-1}$. One simply observes that there is an obvious bound on the conjugates of $B_{n-1}(\beta)$ owing to the fact that the other conjugates of $\beta$ all have absolute value $\leq 1$, and that the product of all the conjugates is a nonzero integer.

In practice, we can simply choose an approximation $\beta_{0}$ to $\beta$ of fixed accuracy $\epsilon$. (We usually chose $\epsilon$ to be $5 \times 10^{-16}$ or $5 \times 10^{-32}$.) The number $\xi=\beta_{0} B_{n-1}\left(\beta_{0}\right)$ is an approximation to $\beta \alpha_{n-1}$ whose accuracy is easily estimated. If $x B_{n-1}(x)=$ $b_{1} x+b_{2} x^{2}+\cdots+b_{d} x^{d}$, and if $\eta=\sum\left|b_{i}\right| i\left(\beta_{0}+\epsilon\right)^{i-1} \epsilon$, then $\left|\beta \alpha_{n-1}-\xi\right|<\eta$. Thus, if the distance from $\xi$ to the nearest integer is at least $\eta$, then $\lfloor\xi\rfloor=\left\lfloor\beta \alpha_{n-1}\right\rfloor=c_{n}$.

Our algorithm begins with $\epsilon=5 \times 10^{-16}$ (double precision). If at some point in the computation, the criterion of the previous paragraph fails, the computation of $\xi$ is repeated with a $\beta_{0}$ having accuracy $\epsilon<5 \times 10^{-32}$ (quadruple precision). If the criterion fails at this level of accuracy, then the computation is terminated. This occurred for only five values of $\beta$ in our entire project, one of which can be recognized in Table 2: $(m, p)$ is not given, but the corresponding lower bound on $D$ is $<10^{6}$.

For each $(a, b, c)$, the values of $n$ for which a change from double precision to quadruple precision was necessary were recorded in a file during the computation. We indicate below how these values were used in some cases to determine some of the larger values of $(m, p)$.

As a sample of the accuracy needed, suppose we were to take $\epsilon=10^{-13}$ for the $\beta$ with $(a, b, c)=(-3,-1,-7)$. Then for $n=167305$ one computes $\xi=1.0000004249$, suggesting that $c_{n}=1$, while in fact $\beta \alpha_{n-1}=.9999999851$, so $c_{n}=0$. On the other hand, for most values of $n$, a far less accurate value of $\beta_{0}$ would suffice. The twotier arrangement described in the previous paragraph allows one to make use of the fast double-precision multiplication on the machine used (an Amdahl 5860) while retaining the benefits of a higher-precision calculation when required. One could easily envision a multiple-tier approach.

The sequence $\left\{\alpha_{n}\right\}$ is periodic if and only if $\left\{L\left(B_{n}\right)\right\}$ is bounded. Thus, if $\beta$ is a beta number, its expansion can be computed using an approximation $\beta_{0}$ of some fixed accuracy (which depends on $\beta$, of course).

5.2. Variations on the basic algorithm. An alternative approach, which avoids the computation of $\eta$ at each step, is to compute $c_{n}$ by using integer arithmetic. Let $M$ be the companion matrix to $P(x)$, so that the eigenvalues of $M$ are the conjugates of $\beta$. If $B_{n}(x)$, as above, is such that $\alpha_{n}=B_{n}(\beta)$, then the eigenvalues of $B_{n}(M)$ are the conjugates of $\alpha_{n}$ and the eigenvalues of $M B_{n}(M)$ are the conjugates of $\beta \alpha_{n}$.

Now, $\beta \alpha_{n}$ has exactly two real conjugates corresponding to the two conjugates $\beta$ and $\beta^{-1}$ of $\beta$. Since $0 \leq c_{k}<\beta$ for all $k$, it is easy to see that the conjugate of 
$\beta \alpha_{n}$ corresponding to $\beta^{-1}$ is $\beta^{-1} B_{n}\left(\beta^{-1}\right)=\beta^{-1} P_{n}\left(\beta^{-1}\right)<0$. Let us denote this conjugate by $\gamma_{n}$. For $c \geq 0, \beta \alpha_{n}-c$ has two real conjugates $\gamma_{n}-c<0$ and $\beta \alpha_{n}-c$ itself. By definition, $c_{n}=\max \left\{c: \beta \alpha_{n}-c>0\right\}$. Since all other conjugates are nonreal, the sign of the product of all conjugates is determined by that of $\beta \alpha_{n}-c$, and thus

$$
c_{n}=\max \left\{c: \operatorname{det}\left(M B_{n}(M)-c\right)<0\right\},
$$

which is a compuation involving only finding the determinant of a finite number of matrices with integer entries.

Note that the matrix $B_{n}(M)$ is as good a representation of $\alpha_{n}$ as is $B_{n}(x)$ and can be computed from the recursion $B_{n+1}(M)=M B_{n}(M)-c_{n}$ without explicitly computing the coefficients of $B_{n}(x)$. Of course, $B_{n}(M)$ has $d^{2}$ integer entries rather than the $d$ entries of the coefficient vector of $B_{n}(x)$.

Since the determinant computed above is just the resultant of $x B_{n}(x)-c$ and $P(x)$, another alternative is to use the coefficient vector of $B_{n}(x)$ to represent $\alpha_{n}$ and replace the determinant computation by the computation of a resultant at each step. Or, combining the approaches of $\S 5.1$ and $\S 5.2$, one could compute $c_{n}$ using floating point except in "delicate" cases.

Experiments showed that the approach of $\S 5.2$ was generally considerably slower than that of $\S 5.1$.

5.3. Detecting periodicity. A standard method for detecting periodicity in single-step recurrences is Floyd's algorithm, described in [6, p.7, exs. 6 \& 7]. Having computed $B_{1}, \ldots, B_{2 n}$, one tests whether $B_{n}=B_{2 n}$. If $\left\{B_{n}\right\}$ is periodic with $B_{m}=B_{m+p}$ for minimal $m$ and $p$, and if $n=s p$ satisfies $m \leq s p<m+p$, then $B_{n}=B_{2 n}$. Once $n=s p$ is found, one determines $p$ by finding the minimal divisor of $n$ for which $B_{n}=B_{n+d}$. Finally, one finds $m$ by testing whether $B_{j}=B_{j+p}$ for $j \leq n$.

For small $N$, one can do this in a straightforward way for $n \leq N$ by storing a table of $B_{1}, \ldots, B_{2 N}$. For larger $N$, once memory becomes insufficient, one instead computes $B_{n}, B_{2 n-1}$ and $B_{2 n}$ at each step. This requires $3 N$ of the basic steps to reach $n=N$, rather than $2 N$.

If we have not proved that the sequence is periodic by the time we have reached $B_{2 N}$, then we know that $D=m+p>N$. This observation accounts for only one of the lower bounds in Table 2 , namely $(-7,-29,-43)$, for which the lower bound is exactly $10^{6}$.

A more useful method for obtaining lower bounds on $m+p$ is based on the following observation. Let $H\left(B_{n}\right)$ denote the maximum of the absolute values of the coefficients of $B_{n}$. During the computation, maintain a list of the record values of $H\left(B_{n}\right)$, i.e., those $n$ for which $H\left(B_{n}\right)>H\left(B_{k}\right)$ for all $k<n$. Clearly, if $H\left(B_{n}\right)$ is a record, then $B_{1}, \ldots, B_{n}$ are all distinct, so we know that $D>n$. In those cases for which a preliminary computation up to $N=10^{5}$, say, had indicated that $\left\{B_{n}\right\}$ did not have a small period, our program continued to compute $B_{n}$ up to $N=2 \times 10^{6}$ without employing Floyd's algorithm. If the last record occurred for $n>10^{6}$, then this was larger than could have been obtained from Floyd's algorithm and the cost was less than $2 / 3$ the cost of using that algorithm. On the other hand, in those rare cases where the last record occurred for $n<10^{6}$, the computation was repeated using Floyd's algorithm and a relatively small $(m, p)$ was usually detected.

For example, with $(a, b, c)=(-9,-23,-28)$, a computation up to $N=10^{9}$ indicated that the last record occurred at $n=1782995$, suggesting a periodic sequence. 
A recomputation of $B_{n}$ in the range $1780000 \leq n<3800000$ found the values $(m, p)=(1979174,11754)$.

As mentioned above, a list was maintained of values of $n, B_{n}$ for which the computation of $c_{n}$ required quadruple precision. Let us call these $n$ markers. Suppose one has computed $B_{1}, \ldots, B_{N}$, where $N>m+2 p$, and that one of these markers, $n_{1}$, occurs within the periodic part of the sequence, that is, for $m \leq n<m+p$. Then, by periodicity, $n_{2}=n_{1}+p$ must be a marker and thus must occur within the list of markers. Thus, by scanning this list of $B_{n}$ for repeats, one can obtain $p$ directly, as well as an upper bound on $m$. A recomputation of $B_{n}$ in a short range enables one to compute $m$ exactly.

For example, with $(a, b, c)=(-6,-5,-14)$, we computed $B_{n}$ up to $N=2 \times 10^{8}$ and determined that the last record occurred for $n=45622056$, suggesting that the sequence was possibly periodic. A check of the list of markers revealed that $B_{n}=B_{n+q}$ for $n=39667761$ and $n+q=132886569$, and that no marker $k$ between these values had $B_{k}=B_{n}$. Thus, the period was revealed to be $p=q=93218808$. Since $n_{1}=39260289$ is a marker, but $n_{1}+q$ is not, the list also showed that $n_{1}<m<n$, and a binary search in this range revealed that $m=39420662$.

On the other hand, for $(a, b, c)=(-3,-1,-7)$, we computed $B_{n}$ up to $N=1.2 \times$ $10^{9}$. The last record of $H\left(B_{n}\right)$ (which was 81363), occurred for $n=1199978517$, giving the lower bound for $D=m+p$ listed in Table 2 . The computation of $10^{9}$ values of $B_{n}$ required 8.25 hours of CPU time on the Amdahl 5860. Even if $p<\infty$, it does not seem practical to compute $p$ in this case by a direct approach unless $p$ should happen to be quite small relative to $m+p$.

In addition to the markers, a table of $B_{n}$ for multiples of $10^{6}$ was also maintained, so that the computation could be restarted from any such value without having to recompute the entire sequence. Although the markers here arose in a natural way from the algorithm employed, one could clearly also use some more artificial criterion for inventing markers. For example, one could store all those pairs $\left(n, B_{n}\right)$ with the first component of $B_{n}$ divisible by 1000 . Then, in a computation up to $N=10^{9}$ one would expect about $10^{6}$ values of $B_{n}$ to be stored, and that one of these values would occur within the period unless $p$ was unusually small.

\section{A PROBABILISTIC MODEL}

6.1. Some ideas from the geometry of numbers. If $\beta$ is a Salem number of degree $d=2 s$, then it has two real conjugates, $\beta_{1}=\beta$ and $\beta_{s+1}=1 / \beta$, and $s-1$ pairs of complex conjugates $\beta_{j}=\overline{\beta_{s+j}}$ for $2 \leq j \leq s$. All the numbers $\alpha_{n}=T^{n}(1)$ lie in the set $\mathbb{Z}(\beta)$. As is familiar from the geometry of numbers [2, p.96], we can think of $\mathbb{Z}(\beta)$ as a lattice $\Lambda$ in $\mathbb{R}^{d}$ defined by mapping $\gamma \in \mathbb{Z}(\beta)$ onto the point $\psi(\gamma)=\left(\gamma_{1}, \Re\left(\gamma_{2}\right), \Im\left(\gamma_{2}\right), \ldots, \Re\left(\gamma_{s}\right), \Im\left(\gamma_{s}\right), \gamma_{s+1}\right)$, where $\gamma_{j}$ denotes the conjugate of $\gamma$ corresponding to the conjugation $\beta \rightarrow \beta_{j}$.

The determinant of $\Lambda\left[2\right.$, p.99] is $\operatorname{det}(\Lambda)=2^{-(d-2) / 2} \sqrt{ }|\operatorname{disc}(\beta)|$, since there are $d-2$ nonreal conjugates, where $\operatorname{disc}(\beta)$ denotes not the discriminant of the field $\mathbb{Q}(\beta)$, but rather the discriminant of the order $\mathbb{Z}(\beta)$ i.e., the discriminant of the minimal polynomial $P$ of $\beta$. Thus, the number of points of $\Lambda$ in a large cube of volume $V$ is asymptotically $V / \operatorname{det}(\Lambda)$.

Now, consider the iterates $\alpha_{n}=T^{n}(1)$ as points in the lattice $\Lambda$. By definition, $0<\alpha_{n}<1$. The conjugate $\delta_{n}$ of $\alpha_{n}$ corresponding to $1 / \beta$ is $\delta_{n}=P_{n}(1 / \beta)$, which satisfies $-\beta^{2} /(\beta-1)<\delta_{n}<0$. For a typical conjugate $\gamma$ with $|\gamma|=1$, 
the corresponding conjugate of $\alpha_{n}$ is $\gamma_{n}=\left|P_{n}(\gamma)\right|<1+\lfloor\beta\rfloor n$. However, it is reasonable to expect that $\gamma_{n}$ may be $O\left(n^{\delta}\right)$ for some $\delta<1$. In fact, it is plausible that $\delta=1 / 2$, as we argue in $\S 6.4$ below. The following result contains the only completely rigorous argument of this section.

Proposition 6.1. Suppose that $\operatorname{deg}(\beta)=d$, and that $\left|\gamma_{n}\right|=O\left(n^{\delta}\right)$ for all conjugates $|\gamma|=1$, for some $\delta<1 / d$. Then $\beta$ is a beta number (and hence $\left|\gamma_{n}\right|=O(1)$ ).

Proof. The points $\alpha_{1}, \ldots, \alpha_{n}$ of the orbit correspond to the points $\psi\left(\alpha_{1}\right), \ldots, \psi\left(\alpha_{n}\right)$ in the lattice $\Lambda$. By the estimates on the conjugates just given, these $n$ points lie in a cube $C_{n}$ of volume $V_{n}=O\left(n^{\delta d}\right)=o(n)$, and since the points of the lattice have a constant density in $\mathbb{R}^{d}$, there are $o(n)$ points of the lattice in $C_{n}$. By the box principle, for sufficiently large $n$ we must have $\alpha_{i}=\alpha_{j}$ for some $i \neq j$ and hence the orbit is finite. Once we know the orbit is finite, the conjugates $\gamma_{n}$ lie in a finite set, and so certainly $\left|\gamma_{n}\right|=O(1)$.

Remark 6.1. It is not clear how one would prove that $\left|\gamma_{n}\right|=O\left(n^{\delta}\right)$ with $\delta<$ 1 for some given $\beta$. The main virtue of Proposition 6.1 is that it shows that there is a threshold for the rate of growth of $\left|\gamma_{n}\right|$ which must be exceeded before nonperiodicity is possible.

6.2. Replacing a cube by a slab. The condition on $\delta$ in Proposition 6.1 can plausibly be replaced by $\delta<1 /(d-2)$. The points $\psi\left(\alpha_{1}\right), \ldots, \psi\left(\alpha_{n}\right)$ actually lie not just in a cube $C_{n}$ but in a slab $S_{n}$ with 2 sides of bounded length, corresponding to the conjugates $\beta$ and $1 / \beta$, and $d-2$ sides of length $O\left(n^{\delta}\right)$ corresponding to the conjugates $|\gamma|=1$. The volume of $S_{n}$ is $O\left(n^{\delta(d-2)}\right)$, which is $o(n)$ if $\delta<1 /(d-2)$. If we assume that $S_{n}$ lies in "general position" with respect to $\Lambda$, then we would expect it to contain $N_{n}=O\left(n^{\delta(d-2)}\right)=o(n)$ points of $\Lambda$. It is possible that the slab $S_{n}$ is tilted in such a way as to contain more than its fair share of points of $\Lambda$, but we regard this as unlikely. This cannot happen with the cube $C_{n}$ used in the proof of Proposition 6.1 and is the reason it was used there. If the estimate $N_{n}=O\left(n^{\delta(d-2)}\right)$ could be established rigorously then, for $d=4$, the argument here could be applied if $\delta<1 / 2$, which is almost what we get from the nonrigorous argument of $\S 6.4$.

6.3. Assuming the orbit is randomly distributed. If we go beyond the box principle used in $\S 6.1$ and $\S 6.2$ and imagine that the points of the orbit $\alpha_{1}, \ldots, \alpha_{n}$ are distributed "randomly" in the slab $S_{n}$, we can allow a larger value of $\delta$. Suppose that $\left|\gamma_{n}\right|=O\left(n^{\delta}\right)$ with $\delta<2 /(d-2)$, so the volume $V_{n}$ of $S_{n}$ satisfies $V_{n}=O\left(n^{\delta(d-2)}\right)$ and hence, as in $\S 6.2, S_{n}$ contains $N_{n}=O\left(n^{\delta(d-2)}\right)=o\left(n^{2}\right)$ points of the lattice $\Lambda$. Now suppose that the points $\psi\left(\alpha_{1}\right), \ldots, \psi\left(\alpha_{n}\right)$ are randomly chosen from these $N_{n}$ points. Then, by the "birthday paradox" we will have $\alpha_{i}=\alpha_{j}$ for some $i \neq j$ with probability tending to 1 as $n \rightarrow \infty$. That is, the probability that $n$ randomly selected points among $N_{n}$ are distinct is

$$
\prod_{k=1}^{n-1}\left(1-k / N_{n}\right) \sim \prod_{k=1}^{n-1} \exp \left(-k / N_{n}\right) \sim \exp \left(-\frac{1}{2} n^{2} / N_{n}\right) \rightarrow 0
$$

as $n \rightarrow \infty$ since $N_{n}=o\left(n^{2}\right)$.

For $d=4$, this argument requires $\delta<1$, which is just short of what can be proved, while for $d=6$, the requirement is $\delta<1 / 2$, which is just short of the 
$\delta=1 / 2$ we obtain in $\S 6.4$ by a "random walk" argument. For $d \geq 8$, however, even the most favorable assumptions would not seem to justify the expectation that the orbit $\left\{\alpha_{k}\right\}$ should be finite.

6.4. A random walk argument to justify $\delta=1 / 2$. Let us try to justify the assumption that $\left|\gamma_{n}\right|=O\left(n^{1 / 2}\right)$. Assume that the digits $c_{n}$ are randomly distributed according to the following distribution: $\operatorname{Pr}\left\{c_{n}=j\right\}=1 / \beta$, for $0 \leq j \leq$ $\lfloor\beta\rfloor-1$ and $\left.\operatorname{Pr}\left\{c_{n}=\lfloor\beta\rfloor\right\}=(1-\mid \beta\rfloor / \beta\right)$. In addition, assume that the $c_{n}$ are independent. Then $E\left(c_{n}\right) \approx \beta / 2, E\left(c_{n}^{2}\right) \approx \beta^{2} / 3$ and $E\left(c_{m} c_{n}\right) \approx \beta^{2} / 12$ if $m \neq n$.

Now

$$
\left|\gamma_{n}\right|=\left|1-c_{1} \gamma^{-1}-\cdots-c_{n} \gamma^{-n}\right|
$$

and

$$
E\left|\sum_{j=1}^{n} c_{j} \gamma^{j}\right|^{2} \approx \beta^{2} n / 12+\left(\beta^{2} / 4\right)\left|\left(\gamma-\gamma^{n+1}\right) /(1-\gamma)\right|^{2} \approx \beta^{2} n / 12,
$$

SO

$$
\left|\gamma_{n}\right| \approx \beta(n / 12)^{1 / 2}
$$

6.5. Consequences of the assumption $\delta=1 / 2$. Let us explore the consequences of the plausible assumption $\delta=1 / 2$. By (6.3), we expect that $\left|\gamma_{n}\right|=O\left(\beta n^{1 / 2}\right)$, where the $O$ constant is uniform in $\beta$. Thus the region $S_{n}$, being the product of two intervals of lengths 1 and $\beta^{2} /(\beta-1)$ and $(d-2) / 2$ disks of radius $O\left(\beta n^{1 / 2}\right)$, has volume $V_{n}=O\left(\beta^{d-1} n^{(d-2) / 2}\right)$. Since the points of $\Lambda$ are distributed with density $1 / \operatorname{det}(\Lambda)=2^{(d-2) / 2} /|\operatorname{disc}(\beta)|^{1 / 2}$, and assuming $S_{n}$ is in general position, there are $N_{n}=O\left(\beta^{d-1} n^{(d-2) / 2} / \operatorname{disc}(\beta)^{1 / 2}\right)$ points of $\Lambda$ in $S_{n}$. If $\operatorname{disc}(\beta)$ is sufficiently large, it is thus possible to push through the argument of $\S 6.2$ for $d=4$ and the birthday paradox argument of $\S 6.3$ for $d=6$ to conclude that the orbit of $\beta$ should be finite.

More precisely, $V\left(S_{n}\right) \approx \beta^{d-1}(\pi n / 12)^{(d-2) / 2}$, so $N_{n} \approx C(\beta) n^{(d-2) / 2}$, where

$$
C(\beta)=\beta^{d-1}(\pi / 6)^{(d-2) / 2} /|\operatorname{disc}(\beta)|^{1 / 2} .
$$

If $\beta$ is large, then the largest terms in $\operatorname{disc}(\beta)$ are the $d-1$ terms involving the conjugate $\beta$; their product is about $\beta^{2(d-1)}$. If the conjugates on the unit circle are nicely distributed, then we would expect the product of terms involving them not to be too small and so "typically" $\operatorname{disc}(\beta) \sim \beta^{2(d-1)}$ and $C(\beta)=O(1)$ from (6.4). Values of $\operatorname{disc}(\beta)$ which are untypically small will lead to large values of $C(\beta)$.

6.6. The expected size of the orbits. Now, let $p_{n} \sim 1-n /\left(C(\beta) n^{(d-2) / 2}\right)$ be the probability that $\alpha_{n} \neq \alpha_{1}, \ldots, \alpha_{n-1}$. Then the probability that the points $\alpha_{1}, \alpha_{2}, \ldots$ are distinct is

$$
\prod_{n}\left(1-\frac{1}{C(\beta) n^{(d-4) / 2}}\right) \begin{cases}=0 & \text { if } d=4 \text { or } 6 \\ >0 & \text { if } d \geq 8 .\end{cases}
$$

This suggests that almost all orbits are finite if $n=4,6$ while a positive proportion are infinite if $n \geq 8$. 
We can distinguish the cases $n=4$ and $n=6$ by considering the expected size $(D=m+p)$ of the orbit. Using the familiar fact that $E(D)=\sum_{n} \operatorname{Pr}\{D>n\}$, we compute

$$
q_{n}=\operatorname{Pr}\{D>n\}=\prod_{k=1}^{n}\left(1-\frac{1}{C(\beta) k^{(d-4) / 2}}\right) .
$$

For $d=4$, we thus have $q_{n}=q_{1}^{n}$, so

$$
E(D)=q_{1} /\left(1-q_{1}\right)=C(\beta)-1 .
$$

On the other hand, if $d=6$, then

$$
-\log q_{n}=\log n / C(\beta)+O(1),
$$

i.e., $q_{n} \sim A n^{-1 / C(\beta)}$, so that

$$
E(D) \begin{cases}<\infty & \text { if } C(\beta)<1 \\ =\infty & \text { if } C(\beta) \geq 1\end{cases}
$$

Remark 6.2. For $d=4$, we have shown rigorously in [3] that $D(\beta) \leq 2 \operatorname{trace}(\beta)-3 \leq$ $2 \beta+3$. The largest value of $D(\beta)$ for $\beta$ with a fixed value of $\operatorname{trace}(\beta)$ is attained by the $\beta$ with minimal polynomial $P(x)=x^{4}-a x^{3}+(2 a-3) x^{2}-a x+1$. By (2.4), $P$ has discriminant $(4 a-1)\left(a^{2}-8 a+20\right)^{2} \sim \beta^{5}$ for large $\beta$, so that from (6.4), $C(\beta) \sim \beta^{1 / 2}$. Thus $(6.7)$ predicts a smaller value for $D(\beta)$ than actually attained but it does predict that large values of $D(\beta)$ occur for small values of $\operatorname{disc}(\beta)$ and vice versa.

Remark 6.3. For $d=6$, the data tends to confirm a direct relationship between the sizes of $C(\beta)$ and $D(\beta)$, although not quite as dramatic as (6.8) would suggest. There are some exceptions as well: for example if $(a, b, c)=(-9,-37,-55)$, then $\operatorname{disc}(\beta)=140682625$ is quite small, so $C(\beta)=6.6956$ is large, but $D(\beta)=531230$ is not exceptionally large. On the other hand, the example $(-3,-1,-7)$ with $D(\beta)>1.2 \times 10^{9}$ only has $C(\beta)=.3342$, which is not very large (although it is the largest value of $C(\beta)$ by far in Table 3, i.e., for $(a, b)=(-3,-1))$. This is perhaps to be expected, given the number of unsupported assumptions we have had to make to arrive at (6.8).

Perhaps the most questionable argument is the deduction of the exponent $\delta=$ $1 / 2$ in $\S 6.4$. Let $\mu(n)$ denote the maximum modulus of the conjugates of $\alpha_{n}$, i.e., the largest modulus of the eigenvalues of the matrix $B_{n}(M)$ discussed in $§ 5.2$. A fit of a power curve to data from the first 20000 values of $\mu(n)$ for the case $(a, b, c)=(-3,-1,-7)$ suggests an exponent $.3<\delta<.4$ for this example. A plot of $\mu(n)$ versus $n$ in this range suggests some sort of random process but perhaps not one well described by the model of $\S 6.4$. Note that, for $d=6$, we only need $\delta<1 / 2$ to apply the birthday paradox argument of $\S 6.3$, so if the growth observed for $n \leq 20000$ were to persist, then $\S 6.3$ would predict a periodic beta expansion for this $\beta$.

To test this, let us consider the behavior of the record values of $H\left(B_{n}\right)$ for large $n$ still for this $\beta$. So, let $M(n)$ denote the largest value of $H\left(B_{m}\right)$ for $m \leq n$. For $n=10^{k}, k=1, \ldots, 9$, we find that

$$
\left[M\left(10^{k}\right): k=1, \ldots, 9\right]=[6,54,172,414,1326,9701,21497,58133,139410] .
$$


Fitting a power curve to these 9 values (by a linear least squares fit to their logarithms) gives a curve $3.26 n^{.54}$, suggesting $\delta>1 / 2$. On the other hand, using only $k=4, \ldots, 9$ gives $\delta \approx .51$, while the data for the 5 values $k=5, \ldots, 9$ is very well fitted by a curve with $\delta \approx .39$. Apparently, this data does not exclude or confirm a growth rate of $n^{1 / 2}$.

Another instructive example is $(-1,-7,-11)$, where $(m, p)=(2438,863)$. Here, $\mu(n)$ is quite well fitted for $n \leq 2000$ by a curve $A n^{\delta}$ with $\delta=.4$. Of course, this cannot hold for $n \rightarrow \infty$ since $\mu(n)$ is ultimately periodic.

Remark 6.4. Our computations have concentrated on the question of whether Salem numbers are beta numbers and not on Schmidt's more general conjecture that, for every Salem number $\beta$ and every rational $x \in[0,1]$, the orbit $\left\{T^{n}(x)\right\}$ should be finite. Our heuristic arguments apply equally well to this conjecture. If $x=p / q$, then the iterates $T^{n}(x)$ lie in $(1 / q) \mathbb{Z}[\beta]$, and hence we need only replace the lattice $\Lambda$ by $(1 / q) \Lambda$ in the arguments of $\S 6.1$ to $\S 6.6$. The only change is that the density of the points of $(1 / q) \Lambda$ in $\mathbb{R}^{d}$ is $q^{d}$ times as large as the density of the points of $\Lambda$. Thus, the qualitative predictions of $\S 6.1$ to $\S 6.4$ are unchanged, i.e., we predict that Schmidt's conjecture is true for Salem numbers of degree 4 and 6 but not for higher degrees. The main change in the more quantitative analysis of $\S 6.5$ and $\S 6.6$ is that $C(\beta)$ must be replaced by $q^{d} C(\beta)$. Thus, $\S 6.6$ would suggest that, for fixed $\beta$, the size of the orbit $\left\{T^{n}(p / q)\right\}$ should increase with $q$ as predicted by (6.7) and (6.8). That is, for $d=4$ the size of the orbit should be roughly proportional to $q^{4}$, and for $d=6$ we should expect unusually large orbits as soon as $q^{6} C(\beta)>1$. It would be interesting to test these predictions by further computation.

\section{REFERENCES}

1. A. Bertrand, Développements en base de Pisot et répartition modulo 1, C.R. Acad. Sci. Paris Sér. I Math. 285 (1977), 419-421. MR 56:5449

2. Z.I. Borevich and I.R. Shafarevich, Number Theory, Academic Press, New York and London, 1966. MR 33:4001

3. D.W. Boyd, Salem numbers of degree four have periodic expansions, Théorie des Nombres Number Theory (J.M. de Koninck and C. Levesque, eds.), Walter de Gruyter \& Co., Berlin and New York, 1989, pp. 57-64. MR 90j:11071

4. L. Flatto, J.C. Lagarias and B. Poonen, The zeta function of the beta transformation, Ergodic Theory Dynamical Systems 14 (1994), 237-266. MR 95c:58141

5. A.O. Gelfond, On a general property of number systems, Izv. Akad. Nauk SSSR Ser. Mat. 23 (1959), 809-814. (Russian) MR 22:702

6. D. Knuth, The art of computer programming, volume 1 : Fundamental algorithms, AddisonWesley, Reading, MA, 1969. MR 44:3530

7. W. Parry, On the $\beta$-expansions of real numbers, Acta Math. Hungar. 11 (1960), 401-416. MR 26:288

8. A. Rényi, Representations for real numbers and their ergodic properties, Acta Math. Hungar. 8 (1957), 477-493. MR 20:3843

9. R. Salem, Algebraic numbers and Fourier analysis, D. C. Heath \& Co., Boston, 1963. MR 28:1169

10. K. Schmidt, On periodic expansions of Pisot numbers and Salem numbers, Bull. London Math. Soc. 12 (1980), 269-278. MR 82c:12003

11. B. Solomyak, Conjugates of beta-numbers and the zero-free domain for a class of analytic functions, Proc. London Math. Soc. (3) 68 (1994), 477-498. MR 95c:30010

Department of Mathematics, University of British Columbia, Vancouver, B.C., CANADA V6T 1 Z2

E-mail address: boyd@math.ubc.ca 